\title{
Tracheobronchopathia Osteochondroplastica: A Case Report
}

\author{
Karhate $\mathbf{M}^{*}$, Senhaji L, Benjeloun MC, Serraj M \\ Department of Pneumology, Hassan II Unversity Hospital, Fes, Morocco
}

*Corresponding author: Karhate M, Department of Pneumology, Hassan II Unversity Hospital, Fes, Morocco, Tel: 212662765080, E-mail: meryem.andaloussi01@gmail.com, meryem.karhate@gmail.com

Citation: Karhate M, Senhaji L, Benjeloun MC, Serraj M (2019) Tracheobronchopathia Osteochondroplastica: A Case Report. J Case Rep Stud 7(2): 203. doi: 10.15744/2348-9820.7.203

Received Date: November 27, 2018 Accepted Date: April 24, 2019 Published Date: April 26, 2019

\begin{abstract}
Background: Tracheobronchopathia osteochondroplastica (TOCP) is a rare disease defined by the presence of osteocartilaginous foci in the sub mucosa, without direct connection to the cartilage structures of the respiratory tree. The disease is usually located in the trachea, sometimes in the bronchi and more rarely in the larynx.

Case presentation: Patient aged 40 years, admitted to an inspiratory dyspnea table evolving for 1 year. The clinical examination found a wheezing on tracheal auscultation with sibilant rales at 2 respiratory times. The chest X-ray finds an irregular narrowing of the tracheal lumen in the lower part of the chest. Thoracic CT showed a thickening of the walls anterolateral tracheal tubes, which extend towards the large bronchi, associated with irregular calcifications of the wall, which contribute to the obstruction of tracheobronchial light. The patient had a rigid EB that returned to a fragile tracheal wall that bleeds easily on contact, with smooth white nodules respecting the posterior membrane. Biopsies were performed causing significant bleeding, which stopped exploration. Anapathic result: A moderately keratotic squamous mucosa, free of cellular atypia and containing dystrophic calcifications indicative of tracheal inflammation.
\end{abstract}

Conclusion: TOCP remains a rare disease but should be considered in the case of an evocative CT scan. It must be confirmed by flexible or rigid bronchoscopy, which shows an evocative appearance and eliminates differential diagnoses. Several therapeutic modalities have been proposed but the reference treatment remains the symptomatic treatment that improves the quality of life of patients

Keywords: Tracheobronchopathia Osteochondroplastica; Calcifications of the Tracheal Wall

\section{Introduction}

Tracheobronchopathia osteochondroplastica (TOCP) is a rare disease defined by the presence of osteocartilaginous foci in the sub mucosa, without direct connection to the cartilage structures of the respiratory tree. The disease is usually located in the trachea, sometimes in the bronchi and more rarely in the larynx $[1,2]$.

It is a benign condition, of unknown etiology, with an unspecific symptomatology leading to a delayed diagnosis. It is an adult disease that affects both men and women.

\section{Observation}

Patient aged 40 years, with no pathological history, admitted to an inspiratory dyspnea table evolving for 1 year associated with a dry cough evolving in a context of conservation of the general state without other respiratory or extra respiratory signs. The clinical examination found a eupenic patient, who was $94 \%$ saturated with ambient air and on pleuro-pulmonary examination had a wheezing on tracheal auscultation with sibilant rales at 2 respiratory times. The rest of the examination was not unusual. ENT examination of the nose and larynx is without particularity.

The chest X-ray finds an irregular narrowing of the tracheal lumen in the lower part of the chest. Thoracic CT showed a thickening of the walls anterolateral tracheal tubes, which extend towards the large bronchi, associated with irregular calcifications of the wall, which contribute to the obstruction of tracheobronchial light (Figure 1).

The patient had a rigid EB that returned to a fragile tracheal wall that bleeds easily on contact, with smooth white nodules respecting the posterior membrane. Biopsies were performed causing significant bleeding, which stopped exploration. Anapathic result: A moderately keratotic squamous mucosa, free of cellular atypia and containing dystrophic calcifications indicative of tracheal inflammation. 

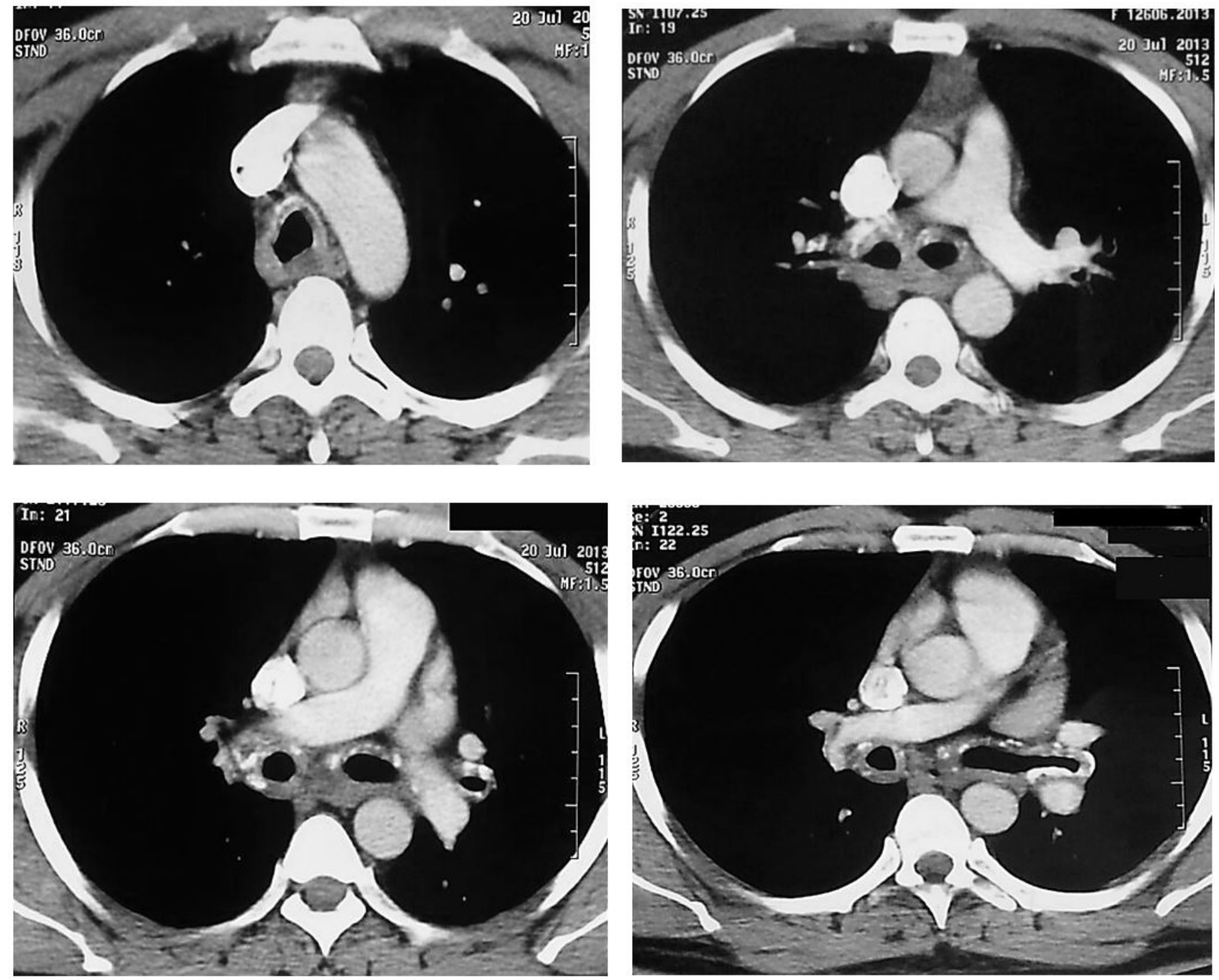

Figure 1: Scan section: Calcifications of the tracheal wall, and of the stem bronchial tubes sparing the posterior wall

\section{Diagnosis}

Tracheobronchopathia osteochondroplastica due to the evocative tomodensitometric and endoscopic aspect.

\section{Discussion}

The first case report of TOCP was described by Wilks in 1857 [3]. It is difficult to estimate the prevalence of TOCP. Bronchoscopy reveals typical lesions in $0.017 \%$ to $0.7 \%$ of cases [4-6]. In the Bioque al study the frequency was $0.11 \%$ [7].

To our knowledge, to date, more than 500 cases of À have been reported worldwide, including about 140 cases in Japanese literature and 80 in Chinese publications [8].

The most important series published is that of Leske, et al., in 2001, which found an equal distribution of patients ( $\mathrm{n}=41$ cases) between the two sexes, with a median age at diagnosis of 63 years and $24 \%$ of smokers [9]. To date, the etiopathogenesis of this disease remains enigmatic. Several hypotheses have been put forward; the most incriminating factors are chronic infections such as Klebsiella ozaenae or chronic irritations [10].

The possibility of a relationship between atrophic rhinitis and TOCP has been raised because these two diseases have the same characteristics of squamous metaplasia in the nasal and tracheal epithelium. However, the prevalence of atrophic rhinitis in patients with TOCP is low [11]. Some authors believe that ossification of tracheopathy is the ultimate evolution of tracheobronchial amyloidosis [1,2].

Leske, et al., detected infectious agents in $61 \%$ of their patients in the respiratory tree with Klebsiella ozaenae and then Pseudomonas aeruginosa as the most frequent germ. Klebsiella ozaenae infection could play a role in the development of TOCP through an experimentally demonstrated property in this germ in particular: its ability to bind directly to the hair cells of the respiratory epithelium inducing ciliostasis and an alteration of mucociliary clearance. For mycobacterial infections, anatomopathological 
analysis eliminates any confusion between tracheal tuberculosis lesions and TOCP, but their coexistence is possible without any direct link ever having been established [12].

Phosphocalcic metabolism abnormalities have long been one of the main pathophysiological hypotheses of TOCP. However, no association between TOCP and a phosphorus and calcium metabolism anomaly or between TOCP and growth hormone hypersecretion has been found [13].

BMP-2 (bone morphogenetic protein-2) could induce de novo bone formation and be involved in the differentiation of mesenchymal cells into cartilage and their mineralization. TGF- $\beta 1$ (transforming growth protein-2) could also play a pathological role; it is detected in chondrocytes, it is found in the tracheal epithelium. There is probably a synergistic action of BMP-2 and TGF would play a role in the formation of bone nodules in the submucosa of TOCP patients, since high levels have been found near cartilage nodules and bone biopsy in TOCP patients $[1,12]$.

The largest series of patients with TOCP has been published by the Group for Studies and Research on "orphan" lung diseases (GERM 0 P). TOCP mainly affects adults over 50 years of age with an average age of 63 years (25 to 85 years) at diagnosis. The sex ratio is equal to one; no family cases are reported. However, lesions probably start earlier due to the long diagnosis time, from 4 to 10 years in the GERM 0 P series [9].

Translated with www.DeepL.com/ Translator There is no relationship between the incidence of the disease and sex or exposure to tobacco $[1,6,14]$. Most patients are asymptomatic for the rest of their lives. The disease is usually discovered by chance during a nasofibroscopy performed for other reasons, or Thoracic CT or difficult intubation [4]. Some authors have reported cases of hemoptysis in patients with TOCP $[1,15]$.

Many patients are misdiagnosed as asthma or chronic bronchitis. While others consult for voice alteration or chronic hoarseness $[1,4]$. Mark, et al., reported cases of TO reveal by middle lobe syndrome [2]. Xi-Qian Xing and al reported a case of TOCP revealed by chest pain [16]. Danckers M, et al., and Hantous, et al., have reported cases of TOCP that were diagnosed upon admission to the intensive care unit for hypercapnic respiratory failure [17-19]. Reza Nikandish reported the case of a 67-year-old male who had a perforation of the trachea by the balloon of the secondary tracheostomy cannula to a TOCP [17]. Nielsen, et al., reported the case of 3 patients who had thyroid and cricoid cartilage damage. One of the patients consulted for dysphonia secondary to TOCP lesions exclusively within the thyroid cartilage [14].

In our observation the patient had inspiratory dyspnea for 1 year. Physical examination is generally normal. Sometimes you can find wheezing, stridor, crepitation. Standard chest X-ray can reveal the diagnosis by showing irregularities and a sinuous aspect of the tracheal lumen. In an advanced stage a narrowing or displacement of the trachea may be visible. Biological tests, particularly the determination of calcium, phosphate and parathyroid are most often normal and have no interest in the diagnosis of the disease $[4,14]$.

Thickening of the anterolateral walls of the trachea and large bronchi, irregular calcifications of the wall, nodules that do not contrast and contribute to the obstruction of the tracheal lumen, the posterior wall of the trachea remains free [11,20]. Lesions may also be visible in the larynx and peripheral bronchi [21]. Finally, the trachea can be totally deformed with a trifoliate aspect as in the observations reported by Saint Blancard, et al., [4,22]. Spirometry is generally normal only a small fraction of patients have an obstructive ventilatory disorder $[2,14,23]$.

It is bronchoscopy that confirms the diagnosis of TOCP. According to some authors, it is used to diagnose 90\% of cases [4,23]. It shows a pathognomonic aspect. The mucosa is deformed by irregular calcifications, more or less confluent white nodules in plaque that can reduce endoluminal size the mucous membrane which is pale, atrophic but never ulcerated. The nodules are of hard consistency, the sensation of squealing during biopsies testifies to the rigidity of the tracheal wall. The posterior wall of the trachea is usually free of pathological lesions and is a symptom that differs TOCP from other respiratory disorders such as amyloidosis. Biopsies are difficult and low cost.

Histological confirmation of the diagnosis is not necessarily necessary, although it may exclude other diseases such as amyloidosis, tumours or papillomas. Especially since sampling most often requires the use of rigid bronchoscopy. It objectifies the presence of cartilage or bone deposits in the chorion, regardless of the normal cartilage structures of the wall, the mucosa is intact [1,4,11,22].

Generally, the evolution is slow, the prognosis remains good because the condition is not very progressive [4].

The lesions do not appear to have malignant potential. Van Nierop, et al., reported one case of TOCP where an endoscopic control performed 8 years after initial assessment showed no progression of lesions [24].

Severe forms can be life-threatening due to an acute respiratory failure pattern. Complications are infectious, mechanical and rarely hemorrhagic $[4,20,25]$. No deaths were attributed to TOCP in the GEMR 0 P series, with a survival of $7 \pm 10$ years [9].

When faced with lesions suggestive of TOCP, the clinician should discuss the relevant differential diagnoses, but avoid using unnecessary additional tests. In general, thickening or stenosis of the cartilage airways should suggest tracheobronchial amylosis, atrophic polychondritis or granulomatous pathology (e. g. Wegener's disease, sarcoidosis, tuberculosis or inflammatory bowel and intestinal diseases). The appearance is not suggestive of a neoplastic pathology. 
The closest pathology to TOCP is tracheobronchial amyloidosis in which $22 \%$ of patients have calcifications. Some even consider TOCP to be an advanced form of it. However, the current trend is to separate TOCP from tracheobronchial amyloidosis from a nosological point of view. During the latter, the CT scan reveals nodules, plaques or circumferential thickening of the trachea and/ or bronchi. The endoscopic aspect is also very different with greyish, friable, sub-mucosal plaques and nodules, bleeding at the slightest contact and not sparing the posterior wall of the trachea. Finally, if performed, biopsies find deposits of amyloid substance in the sub mucosa, revealed by Congo red. Bone metaplasia is very rare.

During atrophic polychondritis, although tracheobronchial involvement may be revealing, the clinical context is often suggestive, including a history of chondrite. CT can then reveal wall calcifications, diffuse and regular thickening of the trachea or bronchi, usually preserving the posterior tracheal wall, and airway collapse malacie on exhalation sections [1].The treatment of TOCP is strictly symptomatic, and there is no curative treatment. It is based on humidification of the respiratory tract, avoidance of exposure to irritants and treatment of respiratory infections $[4,14]$.

Some authors have reported an improvement after administration of bronchodilators [8]. Zhu, et al. suggested the effectiveness of inhaled corticosteroids on nodules that are still in the inflammation stage and have not reached the ossification stage [8]. Hinrich $\mathrm{W}$ reported the case of a patient with a chronic cough with recurrent hemoptysis who improved after inhaling budesonide (200ug) [26].

Li SY, et al., reported one case in which symptoms and mucosal hyperhemia were improved after 6 months of treatment with beclomethasone dipropionate and theophylline [27]. Nodular resection during flexible or rigid bronchoscopy has been proposed in cases of severe airway obstruction.

Dutau and Musani consider interventional bronchoscopy as a highly effective and minimally invasive treatment option [28]. Bioque, et al., suggests that treatment by interventional endoscopy should be reserved for difficult conditions, complicated by hemorrhage, severe stenosis or recurrent airway stenosis [7].

Other methods have been proposed, in particular the implantation of stents in narrowed areas or ablation of nodules by laser and cryotherapy $[2,4]$. HR Jabbardarjani, et al., reported significant clinical and functional improvement by spirometry in patients who received ablation of calcified nodules by laser bronchoscopy [15]. Surgery takes place in localized forms [29]. In some patients, an improvement has been achieved with radiotherapy [4].

\section{Conclusion}

Our patient's profile was similar to the case reported in the literature. TOCP remains a rare disease but should be considered in the case of an evocative CT scan. It must be confirmed by flexible or rigid bronchoscopy, which shows an evocative appearance and eliminates differential diagnoses. Several therapeutic modalities have been proposed but the reference treatment remains the symptomatic treatment that improves the quality of life of patients

\section{References}

1. Samson M, Jibbaoui A, Foucher P, de la Vega MF, Camus P, et al. (2010) Hemoptysis in a smoker revealing an osteochondroplastic tracheobronchopathy (Hémoptysie chez un fumeur révélant une trachéobronchopathie ostéochondroplastique). Med Press 39 : 151-3.

2. Hodges MK, Israel E (1988) Tracheobronchopathia Osteochondroplastica Presenting as Right Middle Lobe Collapse Diagnosis by Bronchoscopy and Computerized tomography. Chest 94: 842-4.

3. Wilks S (1857) Transactions of the Pathological Society of London. London 8: 88.

4. Porzezińska M, Janowicz A, Janowiak P, Cynowska B, Sternau A, et al. (2015) Tracheobronchopathia osteochondroplastica-case report and literature review. Pneumonol Allergol 83: 135-9.

5. Jindal S, Nath A, Neyaz Z, Jaiswal S (2013) Tracheobronchopathia osteochondroplastica-a rare or an overlooked entity? J Radiol Case Rep 7: 16-25.

6. Jabbardarjani HR, Radpey B, Kharaian S, Masjedi MR (2008) Tracheobronchopathia osteochondroplastica: presentation of ten cases and review of the literature. Lung 186: 293-7.

7. Bioque C, Feu N, Rubio JM, Martin MA, Garcia FL, et al. (2001) Tracheobronchopathia osteochondroplastica: clinical study and follow-up in nine cases. J Bronchol Pulmonol 8: 78-83.

8. Zhu Y, Wu N, Huang H-D, Dong Y-C, Sun Q-Y, et al. (2014) A Clinical Study of Tracheobronchopathia Osteochondroplastica: Findings from a Large Chinese Cohort. PLoS ONE 9: e102068.

9. Leske V, Lazor R, Coetmeur D, Crestani B, Chatte G, et al. (2001) Tracheobronchopathia osteochondroplastica: a study of 41 patients. Med (Baltimore) 80: 378-90. 10. Park SS, Shin DH, Lee DH, Jeon SC, Lee JH, et al. (1995) Tracheopathia osteoplastica simulating asthmatic symptoms. Diagnosis by bronchoscopy and compute rized tomography. Respiration 62: 43-5.

11. Ferreira, Morel H, Dixmier A, Arbion F, Marchand-Adam S (2018) Osteochondroplastic tracheobronchopathy: about 3 cases (Trachéobronchopathie ostéochondroplastique : à propos de 3 cas). J Respi Dis 36: 738-44.

12. Wang N, Long F, Jiang S (2016) Tracheobronchopathia osteochondro-plastica: two case reports and review of literature. Med 95: e3396.

13. Nielsen SW, Stevens JR, Dion GR, Howard NS (2015) Dyspnea, Dysphonia, and Cough: Varied Presentations of Tracheobronchopathia Osteochondroplastica. Ann Otol Rhinol Laryngol 124: 829-33.

14. Jabbardarjani H, Radpey B, Kharabian S, Masjedi M (2008) Tracheobronchopathia Osteochondroplastica: Presentation of Ten Cases and Review of the Literature. Lung 186: 293-7. 
15. Xing XQ, Liu YH, Li YL, Zhang HY, Zhang YX, et al. (2015) Tracheobronchopathia Osteochondroplastica: Imaging and Bronchoscopic Findings. Ann Thorac Surg 99: e97.

16. Nikandish R, Fallahi MJ, Ziaian B, Iranpour P (2015) Repeated Tracheostomy Tube Cuff Rupture Due to Tracheobronchopathia Osteochondroplastica: A Case Report. Iran J Otorhinolaryngol 27: 387-90.

17. Danckers M, Raad RA, Zamuco R, Pollack A, Rickert S, et al. (2015) A Complication of Tracheobronchopathia Osteochondroplastica Presenting as Acute Hypercapnic Respiratory Failure. Am J Case Rep 16: 45-9.

18. Hantous-Zannad S, Sebai L, Zidi A, Ben khelil J, Mestiri I, et al. (2003) Tracheobronchopathia osteochondroplastica presenting as a respiratory insufficiency: diagnosis by bronchoscopy and MRI. Eur J Radiol 45: 113-6.

19. Tibesar RJ, Edell ES (2003) Tracheopathia osteoplastica: effective long-term management. Otolaryngol Head Neck Surg 129: 303-4.

20. De wazières B, Fest T, Dalphin JC, Ranfaing, Capellier G, et al. (1994) Tracheopathia osteoplastica associated with dermatomyositis. Case report. Internal Med J 15: 121-3.

21. Saint blancard P, Natali F, Valeyt F, Coutant G, L' Her P, et al. (1997) Osteochondroplastic tracheobronchopathy: five observations (Trachéobronchopathie ostéochondroplastique : cinq observations). Internal Med Dis 18: 882-7.

22. Lundgren R, Stjernberg NL (1981) Tracheobronchopathia osteochondroplastica. A clinical bronchoscopic and spirometric study. Chest 80: 706-9.

23. Van Neirop MA, Wagenaar SS, Van den Bosch JM, Westermann CJ (1983) Tracheobronchopathia osteochondroplastica: report of four cases. Eur J Respir Dis 64: 129-33.

24. Bachy A, Saroul N, Darcha C, Bellini R, Mom T, et al. (2012) An unusual cause of tracheal narrowing: diagnosis and management? (Une cause inhabituelle de rétrécissement trachéal : diagnostic et prise en charge ?) Ann Otorhinolaryngol Head Neck Pathol 129: 246-9.

25. Willms H, Wiechmann V, Sack U, Gillissen A (2008) Tracheobronchopathia osteochondroplastica: A rare cause of chronic cough with haemoptysis. Cough 4: 4. 26. Li S, Ouyang N, Zhong N (2001) Tracheobronchopathia osteochondroplastica. Zhonghua Jie He He Hu Xi Za Zhi 24: 414-6.

27. Dutau H, Musani A (2004) Treatment of severe tracheobronchopathia osteochondroplastica. J Bronchol Interventional Pulmonol 11: 182-18.

28. Liétin B, Vellin JF, Bivahagumye L, Aumaitre O, kemeny JL, et al. (2008) Tracheopathia osteoplastica Trachéopathie ossifiante. Ann Otorhinolaryngol Cervicofac 125: 208-12.

29. Tadjeddein A, Khorgami Z, Akhlaghi H (2006) Tracheobronchopathia osteoplastica: cause of difficult tracheal intubation. Ann Thorac Surg 81: 1480-2. 